\title{
Line Broadening Cross Sections for the Broadening of Transitions of Neutral Atoms by Collisions with Neutral Hydrogen
}

\author{
P. S. Barklem, S. D. Anstee and B. J. O'Mara \\ Department of Physics, University of Queensland, \\ St Lucia, Qld 4072, Australia \\ barklem@physics.uq.edu.au, omara@physics.uq.edu.au \\ Received 1998 February 16, accepted 1998 August 17
}

\begin{abstract}
Line broadening cross sections for the broadening of spectral lines by collisions with neutral hydrogen atoms have been tabulated by Anstee \& O'Mara (1995), Barklem \& O'Mara (1997) and Barklem, O'Mara \& Ross (1998) for s-p, $\mathrm{p}-\mathrm{s}, \mathrm{p}-\mathrm{d}, \mathrm{d}-\mathrm{p}, \mathrm{d}-\mathrm{f}$ and $\mathrm{f}-\mathrm{d}$ transitions. To make these data more accessible to the end user, fortran code which interpolates in these tabulations has been prepared and placed on the World Wide Web. It should be easy to incorporate this code into existing spectrum synthesis programs or to use it in a stand-alone mode to compute line broadening cross sections for specific transitions. The use of the code is demonstrated by its application to two transitions of astrophysical interest.
\end{abstract}

Keywords: atomic data - spectral line profiles

\section{Introduction}

In cool stars such as the sun, the photospheric temperature is sufficiently high to dissociate molecular hydrogen into atomic hydrogen but is insufficient to ionise hydrogen. The electrons present are produced by ionisation of metals leading to an electron number density which is about four orders of magnitude lower than the hydrogen atom density. As hydrogen atoms are the dominant particle species, the collisional broadening of most metallic lines is dominated by collisions with hydrogen atoms. To synthesise spectral lines in cool stars, particularly those with well developed damping wings, it is essential to have satisfactory line broadening data. For the last fifty years astrophysicists have used the broadening theory of Lindholm (1942) and Foley (1946) in a form presented by Unsöld (1955) which assumes a van der Waals interaction between the absorbing atom and the perturbing hydrogen atom. This theory has long been recognised as totally inadequate (see for example Holweger \& Müller 1974). Enhancement factors over the van der Waals broadening, which in general range anywhere from 1 to 4 , have been used by a number of workers to fit solar spectral lines. One of the main reasons that the use of this theory has persisted is the lack of a suitable alternative that is easily used and is applicable to many spectral lines.

Anstee \& O'Mara (1995), Anstee, O'Mara \& Ross (1997), Barklem \& O'Mara (1997) and Barklem, O'Mara \& Ross (1998) have shown that the general theory of broadening developed by Anstee \& O'Mara (1991) leads to solar abundances of certain elements which are consistent with their meteoritic values to within the uncertainties involved, making this theory a natural successor to van der Waals theory. Our aim is to make the determination of line broadening cross sections from this theory even more accessible to users by means of fortran code available over the World Wide Web. More detailed and possibly more accurate calculations of broadening parameters have been undertaken by others, however usually on a line by line basis.

\section{Theory Outline}

The line broadening theory used has been developed from the initial work on the sodium D lines by Anstee \& O'Mara (1991), which itself is a development of the work of Brueckner (1971) and O'Mara (1976). The theory has been further extended to general transitions by Anstee \& O'Mara (1995), Barklem \& O'Mara (1997) and Barklem, O'Mara \& Ross (1998). The theory is well described in these papers, and so here only a brief review is presented.

Collisions with hydrogen atoms are sufficiently fast for the impact approximation of collision broadening to be applicable. This approximation results in a Lorentz profile for the line. For a hydrogen atom number density $N$, and temperature $T$, the line has a half width at half maximum given by

$$
w=N \int_{0}^{\infty} v f(v) \sigma(v) d v,
$$

where $\sigma$ is the line broadening cross section, and $f(v)$ is the Maxwellian distribution of velocities for the given temperature. In this work the perturber trajectory is treated classically, and hence the semiclassical cross section is defined by 


$$
\sigma(v)=2 \pi \int_{0}^{\infty} \operatorname{Re}\left(\langle\Pi(b, v)\rangle_{\mathrm{av}}\right) b \mathrm{~d} b,
$$

where $b$ is the impact parameter defining the distance of closest approach of the atoms, and the line broadening efficiency factor is the real part of a complex efficiency factor $\langle\Pi(b, v)\rangle_{\text {av }}$ averaged over all orientations of the perturbed atom. The efficiency factor $\langle\Pi(b, v)\rangle_{\text {av }}$ can be expressed in terms of the $S$-matrix for the collision, where the $S$-matrix is dependent on the interaction potentials between the atoms. Most broadening theories follow this treatment with the only difference being the method of finding the interaction potential.

In the theory developed by Anstee \& O'Mara (1991) spin is neglected and Rayleigh-Schrödinger perturbation theory taken to second order is used to calculate the interaction energy. The perturbed atom is simply modelled as an atomic core with overall unit charge, with a single valence electron. The Unsöld approximation (1955) is used to simplify the second order expression. Anstee \& O'Mara (1991) have shown that line broadening is dominated by interactions at intermediate separations making perturbation theory particularly appropriate. This is a very important result with implications for all line broadening theories.

The main advantage of the method is that calculations can be made for general states of the perturbed atoms, without requiring any prior knowledge of the species of the atom or energies of states. The potentials may be calculated using Coulomb wavefunctions for the perturbed atom, simply dependent on the effective principal quantum numbers and azimuthal quantum numbers of the two levels of the transition. Anstee \& O'Mara (1995) have tabulated line broadening cross sections for s-p and p-s transitions, Barklem \& O'Mara (1997) for $\mathrm{p}-\mathrm{d}$ and $\mathrm{d}-\mathrm{p}$ transitions and Barklem, O'Mara \& Ross (1998) for $\mathrm{d}-\mathrm{f}$ and $\mathrm{f}-\mathrm{d}$ transitions. In all of these cases, line broadening cross sections were tabulated against effective principal quantum numbers of the upper and lower states for a perturber velocity of $v_{0}=10^{4} \mathrm{~m} \mathrm{~s}^{-1}$. Cross sections were calculated for a range of velocities and were found to obey the velocity law

$$
\sigma(v)=\sigma\left(v_{0}\right)\left(\frac{v}{v_{0}}\right)^{-\alpha}
$$

The parameter $\alpha$ was determined by regression and similarly tabulated.

For cross sections obeying this relationship, Anstee \& O'Mara (1995) found that the linewidth per unit hydrogen atom density could be expressed as

$$
\frac{w}{N}=\left(\frac{4}{\pi}\right)^{\alpha / 2} \Gamma\left(\frac{4-\alpha}{2}\right) v_{0} \sigma\left(v_{0}\right)\left(\bar{v} / v_{0}\right)^{1-\alpha},
$$

where $\bar{v}=(8 k T / \pi \mu)^{\frac{1}{2}}$, and $\mu$ is the reduced mass of the two atoms.

In order to make these data more accessible, fortran code has been written to interpolate these tables. The code simply requires the effective principal quantum numbers and the azimuthal quantum numbers of the upper and lower levels of the transition. Code is also included to compute the linewidth per unit hydrogen atom density from the data, given the temperature.

The code is suitably broken into subroutines which should allow easy integration into other programs if necessary. The program has been successfully tested under DEC fortran. The code uses a bicubic spline interpolation routine from the Numerical Recipes package (Press et al. 1992) for the interpolation of tables. The Gamma function routine is from the Netlib archive (http://netlib.belllabs.com/netlib/master/readme.html).

The code is available on the World Wide Web at http://www.physics.uq.edu.au/people/barklem/ barklem.html. The code can also be obtained by contacting the authors.

It should be noted that this program was not used for the examples given in the papers cited above. These were interpolated using a Matlab program. There may be small differences between the two methods of interpolation.

\section{Examples}

In order to demonstrate how the data are used a brief example is presented. Consider the line of neutral magnesium at $8806 \cdot 76 \AA$. The first step is to identify the levels involved in the transition. Moore's (1972) multiplet tables show this line is from multiplet 7 , with level designations $3^{1} P^{0}-3^{1} D$. Moore's (1971) energy level tables are now consulted to determine the type of transition and the energies of the levels. The $3^{1} P^{0}$ level has an electron configuration of $3 s\left({ }^{2} S\right) 3 p$ and the $3^{1} D$ has an electron configuration of $3 s\left({ }^{2} S\right) 3 d$. The transition therefore is $\mathrm{p}-\mathrm{d}$. The $\mathrm{p}-$ and d-state energies are quoted in wavenumber units as $35051 \cdot 36 \mathrm{~cm}^{-1}$ and $46403 \cdot 14 \mathrm{~cm}^{-1}$ respectively. The effective principal quantum numbers can then be calculated from the formula

$$
n^{*}=\sqrt{\frac{109678 \cdot 8}{\left(E_{\text {limit }}-E_{n l}\right)}},
$$

with all energies in $\mathrm{cm}^{-1}$.

For magnesium the series limit $E_{\text {limit }}$ is $61669 \cdot 14$ $\mathrm{cm}^{-1}$. Hence the effective principal quantum numbers for the $\mathrm{p}$ - and d-states are 2.030 and $2 \cdot 680$ respectively. Using the fortran code previously mentioned the cross section for a relative collision speed of $10^{4} \mathrm{~m} \mathrm{~s}^{-1}$ is found to be 530 atomic units and the velocity parameter is $0 \cdot 277$. The program 
calculates a width per unit hydrogen atom density of $1 \cdot 50 \times 10^{-8} \mathrm{~cm}^{-3} \mathrm{rad} \mathrm{s}^{-1}$ for the hydrogen broadening of the line at $5000 \mathrm{~K}$. Barklem (1998) has shown that this cross section leads to a solar abundance of magnesium in accord with the meteoritic value to within the uncertainty in the $f$-value for the line.

\subsection{Two Electron Excitations}

On some rare occasions, one may have to deal with a transition involving states in which two electrons are excited. What this means, in essence, is that one of the core electrons is also in an excited state during the transition and that our simple model of a single electron outside a singly charged core is no longer strictly applicable. However, as the line broadening cross section is largely determined by the tail of the wave function for the optical electron, useful results can still be obtained provided one is careful in the determination of the appropriate binding energy for the optical electron. As an example, we choose the E-line of Fe I at $5269 \cdot 34 \AA$, first observed by Fraünhofer in the solar spectrum. From Moore's (1972) multiplet tables this transition is from multiplet 15 , with level designations $a^{5} F-z^{5} D^{0}$ with corresponding electron configurations $3 d^{7}\left(a^{4} F\right) 4 s^{-}$ $3 d^{6} 4 s\left(a^{5} D\right) 4 p$. The parent configuration for the upper state corresponds to the ground state of Fe II and therefore the effective principal quantum number is calculated in the usual way. However, the lower state parent configuration $3 d^{7}\left(a^{4} F\right)$ corresponds to the first excited state of Fe II at an energy of $1872 \cdot 60 \mathrm{~cm}^{-1}$ and consequently this energy must be added to the series limit prior to calculating the effective principal quantum number. This leads to an effective principal quantum number for the lower s-state of the transition of 1.368 and an effective principal quantum number of 1.703 for the upper p-state. From the fortran code the cross section for a perturber velocity of $10^{4} \mathrm{~m} \mathrm{~s}^{-1}$ is 237 atomic units and the velocity parameter is $0 \cdot 249$. Anstee, O'Mara \& Ross (1997) have shown that this cross section leads to a solar abundance of iron in excellent agreement with the meteoritic value.

\section{Conclusions}

A program enabling easy interpolation of previously published line broadening data has been demonstrated by application to two lines in the solar spectrum. The source code for this program is available from the World Wide Web. The code is only applicable to lines originating from neutral atoms. We are currently investigating the application of the theory to lines originating from atoms in ionised states.

\section{References}

Anstee, S. D., \& O'Mara, B. J. 1991, MNRAS, 253, 549

Anstee, S. D., \& O'Mara, B. J. 1995, MNRAS, 276, 859

Anstee, S. D., O'Mara, \& B. J., Ross, J. E. 1997, MNRAS, 284, 202

Barklem, P. S. 1998, PhD Thesis, University of Queensland

Barklem, P. S., \& O'Mara, B. J. 1997, MNRAS, 290, 102

Barklem, P. S., O'Mara, B. J., \& Ross, J. E. 1998, MNRAS, 296, 1057

Brueckner, K. A. 1971, ApJ, 169, 621

Foley, H. M. 1946, Phys. Rev., 69, 616

Holweger, H., \& Müller, E. A. 1974, Solar Phys., 3919

Lindholm, E. 1942, PhD Thesis, Uppsala University, Sweden

Moore, C. E. 1971, Atomic energy levels as derived from the analyses of optical spectra (Washington: National Bureau of Standards)

Moore, C. E. 1972, A multiplet table of astrophysical interest (Washington: National Bureau of Standards)

O'Mara, B. J. 1976, MNRAS, 177, 551

Press, W. H., Teukolsky, S. A., Vetterling, W. T., \& Flannery, B. P. 1992, Numerical Recipes in Fortran: The Art of Scientific Computing (Cambridge University Press)

Unsöld, A. 1955, Physik der Stern Atmosphären (Zweite Auflage) (Berlin: Springer) 